\title{
A New Approach for High-Dimensional Unsupervised Learning: Applications to Image Restoration
}

\author{
Nizar Bouguila and Djemel Ziou \\ Département d'Informatique, Faculté des Sciences, \\ Université de Sherbrooke, \\ Sherbrooke, Qc, Canada J1K 2R1 \\ \{nizar.bouguila, djemel.ziou\}@usherbrooke.ca
}

\begin{abstract}
This paper proposes an unsupervised algorithm for learning a high-dimensional finite generalized Dirichlet mixture model. The generalized Dirichlet distribution offers high flexibility and ease of use. We propose a hybrid stochastic expectation maximization algorithm (HSEM) to estimate the parameters of the generalized Dirichlet mixture. The performance of our method is tested by applying it to the problems of image restoration.
\end{abstract}

\section{Introduction}

High-dimensional data appears in many areas such as pattern recognition, computer vision and signal processing. In problems involving the detection of realworld objects, for example, very high-dimensional feature vectors are necessary. In these conditions, use of probabilistic approaches is always delicate. They suffer from a limitation known as the curse of dimensionality. To avoid problems of dimensionality, the most common approaches involve the use of dimensionality reduction, which has been the subject of much research for the past few decades. A good overview of dimensionality reduction techniques is available in [1].

The approach presented in this paper is not aimed at dimensionality reduction. The data is transformed in such a way that density estimation in the transformed space is simpler and more accurate. This transformation is possible thanks to the convenient mathematical proprieties of the generalized Dirichlet density. In previous works, we have shown that the Dirichlet 2 distribution can be good choices to overcome the disadvantages of the Gaussian. Despite its flexibility, the Dirichlet distribution has a very restrictive negative covariance structure. In this paper, we present a generalization of the Dirichlet distribution which has a more general covariance structure than the Dirichlet distribution. We propose a hybrid stochastic expectation maximization (HSEM) algorithm which is a generalization of the EM algorithm. The algorithm is called stochastic because it contains a step in which the data elements are randomly assigned to components in order to avoid convergence to a saddle point. The adjective "hybrid" is justified by the introduction of a Newton-Raphson step. Moreover, 
the HSEM algorithm allow high-dimensional probabilistic modeling. The number of clusters is determined by the introduction of an agglomerative term.

\section{Learning the Finite Generalized Dirichlet Mixture}

If the random vector $\boldsymbol{X}=\left(X_{1}, \ldots, X_{\text {dim }}\right)$ follows a generalized Dirichlet distribution the joint density function is given by [3]:

$$
p\left(X_{1}, \ldots, X_{d i m}\right)=\prod_{i=1}^{\operatorname{dim}} \frac{\Gamma\left(\alpha_{i}+\beta_{i}\right)}{\Gamma\left(\alpha_{i}\right) \Gamma\left(\beta_{i}\right)} X_{i}^{\alpha_{i}-1}\left(1-\sum_{j=1}^{i} X_{j}\right)^{\gamma_{i}}
$$

for $\sum_{i=1}^{\operatorname{dim}} X_{i}<1$ and $0<X_{i}<1$ for $i=1 \ldots d i m$, where $\gamma_{i}=\beta_{i}-\alpha_{i+1}-\beta_{i+1}$ for $i=1 \ldots$ dim -1 and $\gamma_{\text {dim }}=\beta_{\text {dim }}-1$. A generalized Finite Dirichlet mixture with $M$ components is defined as: $p(\boldsymbol{X} / \Theta)=\sum_{j=1}^{M} p\left(\boldsymbol{X} / j, \boldsymbol{\Theta}_{j}\right) P(j)$, where the $P(j)$ are the mixing probabilities and $p\left(\boldsymbol{X} / j, \boldsymbol{\Theta}_{j}\right)$ is the generalized Dirichlet distribution. Each $\boldsymbol{\Theta}_{j}=\left(\alpha_{j 1}, \beta_{j 1}, \ldots, \alpha_{j d i m}, \beta_{j d i m}\right)$ defines the $j$-th component, and $\Theta=\left(\boldsymbol{\Theta}_{1}, \ldots, \boldsymbol{\Theta}_{M}, P(1), \ldots, P(M)\right)$ is the complete set of parameters needed to specify the mixture. Given a set of $N$ independent vectors $\mathcal{X}=\left(\boldsymbol{X}_{1}, \ldots, \boldsymbol{X}_{N}\right)$, the log-likelihood corresponding to a $M$-component mixture is:

$$
L(\Theta, \mathcal{X})=\log \prod_{i=1}^{N} p\left(\boldsymbol{X}_{\boldsymbol{i}} / \Theta\right)=\sum_{i=1}^{N} \log \sum_{j=1}^{M} p\left(\boldsymbol{X}_{i} / j, \boldsymbol{\Theta}_{j}\right) P(j)
$$

It's well-known that the ML estimate: $\hat{\Theta}_{M L}=\operatorname{argmax}_{\Theta}\{L(\Theta, \mathcal{X})\}$. The maximization defining the ML estimates are under constraints $(0<P(j)<1$ and $\left.\sum_{j=1}^{M} P(j)=1\right)$. Obtaining ML estimates of the mixture parameters are possible through EM and related techniques [4. The EM algorithm produces a sequence of estimate $\left\{\Theta^{t}, t=0,1,2 \ldots\right\}$ by alternatingly applying two steps:

1. E-step: Compute the a posteriori probabilities $p\left(j / \boldsymbol{X}_{\boldsymbol{i}}, \boldsymbol{\Theta}_{j}\right)$.

2. M-step: Update the parameters estimates according to: $\hat{\Theta}=\operatorname{argmax}_{\Theta} L(\Theta, \mathcal{X})$

In our approach, we determine the appropriate number of components and the parameters of each component simultaneously by using an agglomerative technique [5. The objective function, using the Lagrange multiplier $\Lambda$ to incorporate the constraints about the mixing parameters, is then:

$$
\Phi(\Theta, \mathcal{X})=\sum_{i=1}^{N} \log \sum_{j=1}^{M} p\left(\boldsymbol{X}_{\boldsymbol{i}} / j, \boldsymbol{\Theta}_{j}\right) P(j)+\Lambda\left(1-\sum_{j=1}^{M} P(j)\right)+\mu \sum_{j=1}^{M} P^{2}(j)
$$

The first term in Eq. 3 is the log-likelihood function, and it assumes its global maximum value when each component represents only one of the feature vectors. The last term (the agglomerative term) reaches its maximum when all of the feature vectors are modeled by a single component, i.e., when $P\left(j_{1}\right)=1$ for some $j_{1}$ and $P(j)=0, \forall j, j \neq j_{1}$. The algorithm starts with an over-specified 
number of components in the mixture, and as it proceeds, components compete to model the data. The update for the a priori probabilities can be shown to be:

$$
P(j)^{(t)}=\frac{\sum_{i=1}^{N} p\left(j / \boldsymbol{X}_{\boldsymbol{i}}, \boldsymbol{\Theta}_{j}\right)^{(t-1)}+2 \mu p^{2}(j)^{(t-1)}}{N+2 \mu \sum_{j=1}^{M} p^{2}(j)^{(t-1)}}
$$

The value of $\mu$ should be chosen such that both terms are of the same order of magnitude. Thus, we choose $\mu$ to be the ratio of the first term to the last term in Eq. 3 of each iteration $t$, i.e.,

$$
\mu(t)=y(t) \frac{\sum_{i=1}^{N} \ln \left(\sum_{j=1}^{M} p^{(t-1)}\left(\boldsymbol{X}_{\boldsymbol{i}} / j, \boldsymbol{\Theta}_{j}\right) P^{(t-1)}(j)\right)}{\sum_{j=1}^{M} P^{2}(j)^{(t-1)}}
$$

The function $y(t)$ is introduced in order to provide a good transition from the non-agglomerative scheme to the agglomerative one. So the value of $\mu$ is increased gradually. The plot of the profile of $y(t)$ versus iteration number is shown in Fig. 1 .

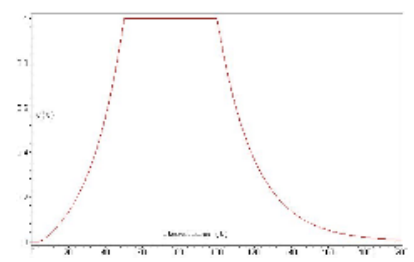

Fig. 1. Plot of the function $\mathrm{y}(\mathrm{t})$ versus iterations $\mathrm{t}$

In order to estimate the $\boldsymbol{\Theta}_{j}$ parameters we will use an interesting property of the generalized Dirichlet distribution. In fact, if a vector $\boldsymbol{X}_{i}$ has a generalized Dirichlet distribution, then we can construct a vector $\boldsymbol{W}_{i}=\left(W_{i 1}, \ldots, W_{i d i m}\right)$ using the following geometric transformation:

$$
W_{i j}=T\left(X_{i j}\right)=\left\{\begin{array}{cc}
X_{i j} & \text { if } j=1 \\
\frac{X_{i j}}{\left(1-\ldots-X_{i j-1}\right)} & \text { for } j=2,3, \ldots, d i m
\end{array}\right.
$$

In this vector $\boldsymbol{W}_{i}$ each $W_{i d}, d=1, \ldots, d i m$ has a Beta distribution with parameters $\alpha_{i d}$ and $\beta_{i d}$ and the parameters $\left\{\alpha_{i d}, \beta_{i d}, d=1, \ldots, d i m\right\}$ define the generalized Dirichlet distribution which $\boldsymbol{X}_{i}$ has 3 . Then, the problem of estimating the parameters of a generalized Dirichlet mixture can be reduced to the estimation of the parameters of $\mathrm{dim}$ Beta mixtures. For this, we must maximize this equation for every dimension:

$$
\Phi_{W}\left(\theta_{d}, \mathcal{W}\right)=\sum_{i=1}^{N} \ln \left(\sum_{j=1}^{M} p_{\text {beta }}\left(W_{i d} / j, \theta_{j d}\right) P(j)\right)
$$

where $\mathcal{W}=\left(W_{1 d}, \ldots, W_{N d}\right), 0<d<d i m, \theta_{d}=\left(\alpha_{1 d}, \beta_{1 d}, \ldots, \alpha_{M d}, \beta_{M d}\right)$, $\theta_{j d}=\left(\alpha_{j d}, \beta_{j d}\right)$ and $P(j)$ are the mixing parameters founded by Eq. 4. The 
maximization of Eq. 7 is equivalent to: $\frac{\partial}{\partial \theta_{j d}} \Phi_{W}\left(\theta_{d}, \mathcal{W}\right)=0 \quad \forall \quad 0<d<$ dim. In order to estimate the $\theta_{j d}$ parameters we have used Fisher's scoring method 6]. The iterative scheme of the Fisher method is given by the following equation:

$$
\left(\begin{array}{c}
\hat{\alpha}_{j d} \\
\hat{\beta}_{j d}
\end{array}\right)^{(t)}=\left(\begin{array}{c}
\hat{\alpha}_{j d} \\
\hat{\beta}_{j d}
\end{array}\right)^{(t-1)}+V^{(t-1)} \times\left(\begin{array}{c}
\frac{\partial \Phi_{W}}{\partial \hat{\alpha}_{j d}} \\
\frac{\partial \Phi_{W}}{\partial \hat{\beta}_{j d}}
\end{array}\right)^{(t-1)}
$$

where $j$ is the class number and $d$ is the current dimension. The matrix $V$ is obtained as the inverse of the Fisher's information matrix. Under sufficiently accurate starting values, the sequence of iterates produced by the Fisher Scoring method enjoys local quadratic convergence to a solution $\hat{\theta}_{j d}$. This rapid convergence can be improved by introducing a Stochastic step in the EM algorithm [7]. In fact, the Stochastic step prevents the sequence of estimates $\Theta^{t}$ from staying near an unstable stationary point of the likelihood function [7]. In order to make our algorithm less sensitive to local maxima, we have used some initialization schemes including the FC-means and the method of moments [2].

INITIALIZATION Algorithm

1. INPUT: $\boldsymbol{X}_{i}, i=1, \ldots, N$ and the number of clusters M.

2. Apply the Fuzzy C-means to obtain the elements, covariance matrix and mean of each component.

3. Compute the $\boldsymbol{W}_{i}=\left(W_{i 1}, \ldots, W_{\text {idim }}\right)$ form the $\boldsymbol{X}_{i}$.

4. Apply the method of moments for each component $j$ and for each dimension $d$ to obtain the vector of parameters $\boldsymbol{\theta}_{j d}$.

5. Assign the data to clusters, assuming that the current model is correct.

6. If the current model and the new model are sufficiently close to each other, terminate, else go to 4 .

With this initialization method in hand, our Hybrid SEM (HSEM) algorithm for estimating of generalized Dirichlet mixture can be summarized as follows:

HSEM Algorithm

1. INPUT: $\boldsymbol{X}_{i}, i=1, \ldots, N$ and an over-specified number of clusters $\mathrm{M}$.

2. Apply the INITIALIZATION Algorithm.

3. E-Step: Compute the a posteriori probabilities:

$$
p\left(j / \boldsymbol{X}_{\boldsymbol{i}}, \boldsymbol{\Theta}_{j}\right)=\frac{p\left(\boldsymbol{X}_{\boldsymbol{i}} / j, \boldsymbol{\Theta}_{j}\right) P(j)}{\sum_{j=1}^{M} p\left(\boldsymbol{X}_{\boldsymbol{i}} / j, \boldsymbol{\Theta}_{j}\right) P(j)}
$$

4. S-Step: For each sample value $\boldsymbol{X}_{i}$ draw $\boldsymbol{Z}_{i}$ from the multinomial distribution of order one with $M$ categories having probabilities specified by the $\hat{Z}_{i j}$.

5. M-Step:

(a) Update the $\boldsymbol{\theta}_{j d}$ using Eq. 8 . $j=1, \ldots, M$. and $d=1, \ldots, d i m$.

(b) Update the $P(j)$ using Eq. 4, $j=1, \ldots, M$.

6. If $P(j)<\epsilon$ discard component $j$, go to 3 .

7. If the convergence test is passed, terminate, else go to 3 . 


\section{Experimental Results: Image Restoration}

Suppose an image has been degraded and we wish to restore it. For this goal, we compare two approaches based on our high-dimensional algorithm and Wiener filtering. We assume that we have a large number of image pairs (original, degraded) for training. In our test, we consider images which are degraded by additive white noise, yet the procedure can be applied for other type of distortion which cannot be expressed simply in mathematical form. For this application, we use an approach proposed in [8. First, vectors are formed for each original pixel in the training set, as shown in Fig. 2, Next, we apply our algorithm to these vectors. After the mixture's parameters are estimated, they can be used to restore a previously unseen degraded test image in the following way. For each pixel location in a given degraded image, let $X_{1}, \ldots, X_{d i m-1}$ denote the observed values of the neighborhood pixels, and let $X_{\text {dim }}$ be the original (unknown) pixel value. The value of $X_{d i m}$ is then chosen according to this rule: $X_{d i m}=\operatorname{argmax}_{X_{d i m}} p\left(\left(X_{1}, \ldots, X_{d i m}\right) / \Theta\right)$. Figure 3 shows the result of generalized Dirichlet mixture-based restoration in an example where two $256 \times 256$ images (see Fig [3, a and Fig 3.e) are degraded by additive white Gaussian noise with a variance of 100 (see Fig [3, b and Fig [3,f). The result of Wiener filtering is

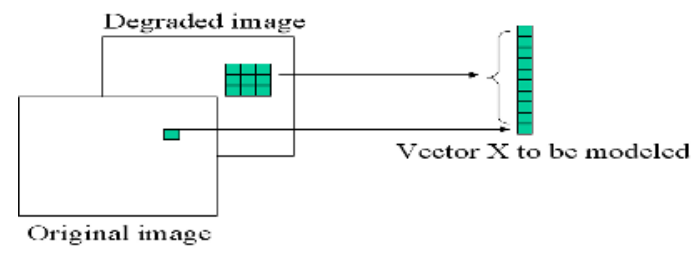

Fig. 2. Formation of data vector $\mathrm{X}$ from degraded and original pixels

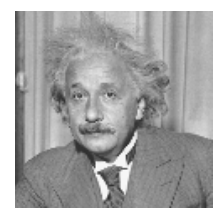

(a)

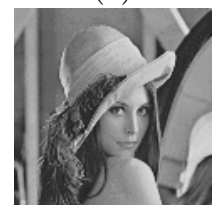

(e)

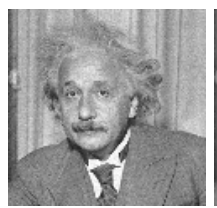

(b)

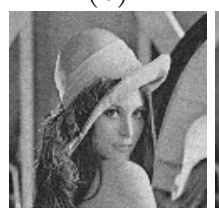

(f)

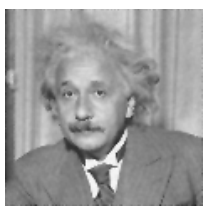

(c)

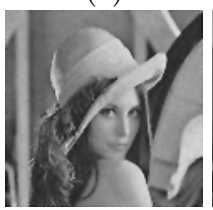

(g)

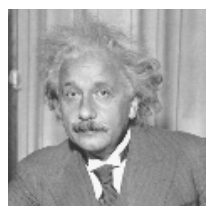

(d)

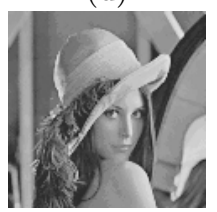

(h)

Fig. 3. Image restoration examples. (a) and (e) original images; (b) and (f) degraded; (c) and (g) Wiener filtered; (d) and (h) restored using generalized Dirichlet mixture. 
shown in figures $3 \mathrm{c}$ and $3 \mathrm{~g}$. For the generalized Dirichlet mixture model a $3 \times 3$ neighborhood was used $(\operatorname{dim}=9+1=10)$. We see clearly that the restored images 3. $\mathrm{d}$ and 3. h exhibits significant noise reduction and maintain good quality. In order to compare the two approaches (Wiener filtering and our algorithm), we have used the SSIM (Structural SIMilarity) index which is a novel method for measuring the similarity between two images and presented by Wang et al. 9]. Then, we applied this method to compare the original images to the restored ones. The SSIM was 0.72 and 0.8 when we compared the original image in Fig 3 ,a with the restored images by the Wiener filtering (see Fig. 3. c) and the restored image by our method (see Fig. 3.d), respectively. We have compared the results given by the two methods for the second images (see Fig. 3. e), too. The wiener filtering and our method gave a SSIM index of 0.74 and 0.78 , respectively.

\section{Conclusion}

In this paper, we have focused on high-dimensional data unsupervised learning. The algorithm proposed is motivated by the great number of pattern recognition and image processing applications which involve such types of data. From the experimental results which involve image restoration, we can say that the generalized Dirichlet distribution offers strong modeling capabilities.

\section{References}

1. S. Aeberhard, D. Coomans and O. De Vel. Comparative Analysis of Statistical Pattern Recognition Methods in High Dimensional Settings. Pattern Recognition, 27(8):1065-1077, 1994.

2. N. Bouguila, D. Ziou and J. Vaillancourt. Unsupervised Learning of a Finite Mixture Model Based on the Dirichlet Distribution and its Application. IEEE Transactions on Image Processing, 13(11):1533-1543, November 2004.

3. R. J. Connor and J. E. Mosimann. Concepts of Independence for Proportions With A Generalization of The Dirichlet Distribution. American Statistical Association Journal, 64:194-206, 1969.

4. G. J. McLachlan and T. Krishnan. The EM Algorithm and Extensions. New York: Wiley-Interscience, 1997.

5. H. Frigui and R. Krishnapuram. A Robust Competitive Clustering Algorithm With Applications in Computer Vision. IEEE Transactions on PAMI, 21(5):450-465, 1999.

6. G.J. McLachlan and D. Peel. Finite Mixture Models. New York: Wiley, 2000.

7. G. Celeux and J. Diebolt. A Stochastic Approximation Type EM Algorithm for the Mixture Problem. Stochastics and Stochastics Reports, 41:119-134, 1992.

8. K. Popat and R. W. Picard. Cluster-Based Probability model and its Application to Image and Texture Processing. IEEE Transactions on Image Processing, 6(2):268284, February 1997.

9. Z. Wang, A. C. Bovik, H. R. Sheikh and E. P. Simoncelli. Image Quality Assessment: From Error Visibility to Structural Similarity. IEEE Transactions on Image Processing, 13(4):600-612, April 2004. 